\title{
Classes of $(0,1)$-matrices where the Bruhat order and the Secondary Bruhat order coincide
}

\author{
Rosário Fernandes · Henrique F. da \\ Cruz • Domingos Salomão
}

Received: date / Accepted: date

\begin{abstract}
Given two nonincreasing integral vectors $R$ and $S$, with the same sum, we denote by $\mathcal{A}(R, S)$ the class of all $(0,1)$-matrices with row sum vector $R$, and column sum vector $S$. The Bruhat order and the Secondary Bruhat order on $\mathcal{A}(R, S)$ are both extensions of the classical Bruhat order on $S_{n}$, the symmetric group of degree $n$. These two partial orders are not, in general, the same. In this paper we prove that if $R=(2,2, \ldots, 2)$ or $R=(1,1, \ldots, 1)$, then the Bruhat order and the Secondary Bruhat order coincide on $\mathcal{A}(R, S)$.
\end{abstract}

Keywords (0,1)-matrices · Partial order · Bruhat order · Secondary Bruhat order

Research partially supported by the projects UID/MAT/04721/2013, and $\mathrm{UID} / \mathrm{MAT} / 00212 / 2013$.

Rosário Fernandes

CMA and Departamento de Matemática, Faculdade de Ciências e Tecnologia, Universidade Nova de Lisboa, 2829-516 Caparica, Portugal.

E-mail: mrff@fct.unl.pt

Henrique F. da Cruz

Departamento de Matemática da Universidade da Beira Interior, Rua Marquês D'Avila e Bolama, 6201-001 Covilhã, Portugal.

E-mail: hcruz@ubi.pt

Domingos Salomão

Universidade Mandume ya Ndemufayo, Escola Superior Pedagógica do Namibe, Angola.

E-mail: d.salomao@ubi.pt 


\section{Introduction}

Let $R=\left(r_{1}, \ldots, r_{m}\right)$, and $S=\left(s_{1}, \ldots, s_{n}\right)$ be two positive integral vectors such that

$$
r_{1}+\ldots+r_{m}=s_{1}+\ldots+s_{n} .
$$

Without loss of generality we assume that $R$ and $S$ are nonincreasing vectors.

If $g_{1}>\ldots>g_{l}$ are positive integers such that $\left\{r_{1}, \ldots, r_{m}\right\}=\left\{g_{1}, \ldots, g_{l}\right\}$, then we also write $R=\left(g_{1}^{i_{1}}, \ldots, g_{l}^{i_{l}}\right)$, where $i_{1}, \ldots, i_{l}$ are the multiplicities of $g_{1}, \ldots, g_{l}$, respectively.

We denote by $\mathcal{A}(R, S)$ the class of all $m$-by- $n$ matrices of zeros and ones, the $(0,1)$-matrices, with row sum vector $R$, and column sum vector $S$. The class of all $n$-by- $n(0,1)$-matrices with common row and column sum $k$ is denoted by $\mathcal{A}(n, k)$.

Let $S_{n}$ be the symmetric group of degree $n$, and let $\sigma \in S_{n}$. We can represent $\sigma$ as a word by $\sigma=\sigma_{1} \ldots \sigma_{n}$, with $\sigma(i)=\sigma_{i}$, for $i=1, \ldots, n$. A pair $(i, j), 1 \leq i, j \leq n$, is called an inversion of $\sigma$ if $i<j$, and $\sigma_{i}>\sigma_{j}$. We can define a partial order $\preceq_{B}$ on $S_{n}$, the Bruhat order, saying $\sigma \preceq_{B} \tau$, if $\sigma$ can be obtained from $\tau$ by a sequence of transformations where

$$
\tau_{1} \ldots \tau_{i} \ldots \tau_{j} \ldots \tau_{n}
$$

is replaced by

$$
\tau_{1} \ldots \tau_{j} \ldots \tau_{i} \ldots \tau_{n}
$$

being $(i, j)$ an inversion of $\tau$.

It is well known that the elements of $S_{n}$ can be represented by matrices, the permutation matrices of order $n$. This allows us to define the Bruhat order in the class of permutation of degree $n$. Note that this class is the class $\mathcal{A}(n, 1)$. Let $P$ and $Q$ be two permutation matrices of order $n$ corresponding to permutations $\pi$ and $\tau$ respectively. We write $P \preceq_{B} Q$ whenever $\pi \preceq_{B} \tau$. Note that a inversion in a permutation $\pi$ corresponds to a submatrix

$$
L_{2}=\left[\begin{array}{ll}
0 & 1 \\
1 & 0
\end{array}\right]
$$

in the correspondent permutation matrix, $P$. Remove this inversion in $\pi$ is equivalent to replace the submatrix $L_{2}$ of $P$ by

$$
I_{2}=\left[\begin{array}{ll}
1 & 0 \\
0 & 1
\end{array}\right] \text {. }
$$

Therefore, the Bruhat order in $\mathcal{A}(n, 1)$ can be interpreted as a sequence of one sided interchanges

$$
L_{2}=\left[\begin{array}{ll}
0 & 1 \\
1 & 0
\end{array}\right] \rightarrow I_{2}=\left[\begin{array}{ll}
1 & 0 \\
0 & 1
\end{array}\right]
$$


There is another way to define the Bruhat order on $\mathcal{A}(n, 1)$. For any $m$ by- $n$ matrix $A=\left[a_{i, j}\right]$ of real entries, let $\Sigma_{A}$ denote the $m$-by- $n$ matrix whose $(r, s)$-entry is

$$
\sigma_{r, s}(A)=\sum_{i=1}^{r} \sum_{j=1}^{s} a_{i j}, \quad 1 \leq r \leq m, 1 \leq s \leq n .
$$

For permutation matrices $P$ and $Q$ of order $n$ is easy to check that $P{ }_{B} Q$ if and only if $\Sigma_{P} \geq \Sigma_{Q}$ by the entrywise order.

Based on this, Brualdi and Hwang extended the Bruhat order from $\mathcal{A}(n, 1)$ to any nonempty classes $\mathcal{A}(R, S)$ (see [3]). Hence, given $A_{1}, A_{2} \in \mathcal{A}(R, S)$ we say that $A_{1}$ precedes $A_{2}$ by the Bruhat order, and write $A_{1} \preceq_{B} A_{2}$ if, by the entrywise order, $\Sigma_{A_{1}} \geq \Sigma_{A_{2}}$. Throughout that paper, [3], it was implicit another partial order relation, but not formally stated. This second order relation was clearly defined a few years later by Brualdi and Deaett in [4]. They called it the Secondary Bruhat order. It states that if $A_{1}, A_{2} \in \mathcal{A}(R, S)$, then we say that $A_{1}$ precedes $A_{2}$ by the Secondary Bruhat order, and write $A_{1} \preceq_{\widehat{B}} A_{2}$, if $A_{1}$ can be obtained from $A_{2}$ by a sequence of one sided interchanges $L_{2} \rightarrow I_{2}$.

These two partial orders have been intensively investigated in the recent years. The research focuses on several topics: minimal elements, [3] and [4], chains and antichains, [10], [9], [15], and [16], restrictions of the Bruhat order to some other classes of $(0,1)$-matrices, [8], and [11], or extensions of the Bruhat order to other classes of matrices than $(0,1)$-matrices, [5], [6], [7], and [13].

It is straightforward to verify that if $A_{1} \preceq \widehat{B} A_{2}$, then $A_{1} \preceq_{B} A_{2}$, that is, the Bruhat order is a refinement of the Secondary Bruhat order. However, in general, the Bruhat order and the Secondary Bruhat order do not coincide in $\mathcal{A}(R, S)$. In fact Brualdi and Deaett proved in [4] that $\preceq_{B}$, and $\preceq_{\widehat{B}}$ coincide in $\mathcal{A}(n, 2)$ as it happens in $\mathcal{A}(n, 1)$ but do not coincide in $\mathcal{A}(6,3)$. Our main goal is to present some more classes where these two partial orders coincide.

This paper is organized as follows. In Section 2 we present some results and techniques needed in squeal. Brualdi and Deaett proved that the Bruhat order and the Secondary Bruhat order are not the same in $\mathcal{A}(6,3)$. In Section 3 we extend this conclusion, proving that the Bruhat order and the Secondary Bruhat order coincide $\mathcal{A}(3,3), \mathcal{A}(4,3)$, and $\mathcal{A}(5,3)$ but they are not the same in $\mathcal{A}(n, 3)$ for $n \geq 6$. In Section 4 where we study the coincidence of these two Bruhat orders in a class $\mathcal{A}(R, S)$ when $r_{1}=\ldots=r_{m}=2$. As a consequence we also prove that these two orders also coincide in a class $\mathcal{A}(R, S)$ when $r_{1}=\ldots=r_{m}=1$. We finish this paper with Section 5 where we present remarks and conclusions.

\section{Auxiliary results}

Throughout we denote by $J_{m, n}$ or simply by $J$ the $m$-by- $n$ matrix whose entries are all equal to one, and by $L_{m}$ we denote the $m$-by- $m$ matrix whose $(i, j)$ entry is one if $i+j=m+1$, and zero otherwise. Given a matrix $A$, the 
submatrix of $A$ that lies in rows $i_{1}, \ldots, i_{r}$ and columns $j_{1}, \ldots, j_{s}$ is denoted by $A\left[\left\{i_{1}, \ldots, i_{r}\right\} ;\left\{j_{1}, \ldots, j_{s}\right\}\right]$.

Let $(X, \leq)$ be a finite partially ordered set. For $a, b \in X$, if $a \neq b$ and $a \leq b$ then we write $a<b$. We say $b$ covers $a$ if $a<b$ and there does not exist $d \in X$ with $a<d<b$.

The cover relation for the Secondary Bruhat order was characterized in [4]. With an elegant result, the authors of [4] show the simplicity of that cover relation.

Theorem 1 [4] Let $A=\left[a_{i, j}\right]$ be a matrix in $\mathcal{A}(R, S)$, where $A[\{i, j\} \mid k, l]=$ $L_{2}$, and let $A^{\prime}=\left[a_{i, j}^{\prime}\right]$ the matrix obtained from $A$ by the $L_{2} \rightarrow I_{2}$ interchange that replaces the submatrix $A[\{i, j\} \mid k, l]=L_{2}$ with $I_{2}$. Then $A$ covers $A^{\prime}$ in the Secondary Bruhat order on $\mathcal{A}(R, S)$ if and only if

1. $a_{p, k}=a_{p, l}, i<p<j$;

2. $a_{i, q}=a_{j, q}, \quad k<q<l$;

3. If $a_{p, k}=a_{i, q}=0$, then $a_{p, q}=0, i<p<j$, and $k<q<l$;

4. If $a_{p, k}=a_{i, q}=1$, then $a_{p, q}=0, i<p<j$, and $k<q<l$.

The following lemmas are used in Section 4. They allow us to identify a submatrix $I_{2}$ in a matrix $A \in \mathcal{A}(R, S)$, with $R=\left(2^{m}\right)$, which is covered by a matrix $C \in \mathcal{A}(R, S)$ by the Bruhat order.

Lemma 1 [4] Let $A$ and $C$ be matrices in $\mathcal{A}(R, S)$ with $A \prec_{B} C$, and let $i$ and $j$ be integers with $\sigma_{i j}(A)>\sigma_{i j}(C)$. Let $s$ and $t$ be integers with $(s, t)$ lexicographically maximal such that

$$
(r, c) \in\{i, \ldots, s-1\} \times\{j, \ldots, t-1\} \Longrightarrow \sigma_{r c}(A)>\sigma_{r c}(C) .
$$

Then, there exists $\left(i_{0}, j_{0}\right) \in\{i+1, \ldots, s\} \times\{j+1, \ldots, t\}$ with $a_{i_{0} j_{0}=1}$

In [8], Lemma 4.3 in [4] was generalized to matrices in $\mathcal{A}(R, S)$.

Lemma 2 [8] Let $A$ and $C$ be matrices in $\mathcal{A}(R, S)$ with $A \prec_{B} C$, and let $i$ and $j$ be integers with $\sigma_{i j}(A)>\sigma_{i j}(C)$. Let $s$ and $t$ be integers with $(s, t)$ lexicographically maximal such that

$$
(r, c) \in\{s+1, \ldots, i\} \times\{t+1, \ldots, j\} \Longrightarrow \sigma_{r c}(A)>\sigma_{r c}(C) .
$$

Then, there exists $\left(i_{0}, j_{0}\right) \in\{s+1, \ldots, i\} \times\{t+1, \ldots, j\}$ with $a_{i_{0} j_{0}=1}$.

Let $A, C \in \mathcal{A}(R, S)$, with $R=\left(2^{m}\right)$. These two lemmas exhibit the nice technique that we used to prove that $A \preceq_{\widehat{B}} C$, when $C$ covers $A$ for the Bruhat order. Our goal is to find a submatrix $I_{2}$ in $A$ such that:

- if we have a position $(i, j)$ in matrix $A$ such that $\sigma_{i j}(A)>\sigma_{i j}(C)$ and $a_{i j}=1$, then we use Lemma 1 , and we conclude that there exists $\left(i_{0}, j_{0}\right) \in$ $\{i+1, \ldots, m\} \times\{j+1, \ldots, n\}$ with $a_{i_{0} j_{0}=1}$, and

for any $(r, c) \in\left\{i, \ldots, i_{0}-1\right\} \times\left\{j, \ldots, j_{0}-1\right\}$ we have $\sigma_{r c}(A)>\sigma_{r c}(C)$. 
- if we have a position $(i, j)$ in matrix $A$ such that $\sigma_{i j}(A)=\sigma_{i j}(C), a_{i j}=1$ and $\sigma_{i-1, j-1}(A)>\sigma_{i-1, j-1}(C)$, then we use Lemma 2 , and we conclude that there exists $\left(i_{0}, j_{0}\right) \in\{1, \ldots, i-1\} \times\{1, \ldots, j-1\}$ with $a_{i_{0} j_{0}=1}$, and for any $(r, c) \in\left\{i_{0}, \ldots, i-1\right\} \times\left\{j_{0}, \ldots, j-1\right\}$ we have $\sigma_{r c}(A)>\sigma_{r c}(C)$.

The following result, which will be used in this paper without comments, was proved in [11] and can be easily checked.

Lemma 3 Let $A=\left[a_{i, j}\right] \in \mathcal{A}(R, S)$. Let $p, f, g$ and $l$ be integers, with $1 \leq p<l \leq m$ and $1 \leq f<g \leq n$, such that

$$
A[\{p, l\} ;\{f, g\}]=L_{2} .
$$

Let $A^{\prime}=\left[a_{i, j}^{\prime}\right]$ be the matrix obtained by $L_{2} \rightarrow I_{2}$ interchange that replaces $A[\{p, l\} ;\{f, g\}]=L_{2}$ with $I_{2}$. Then

$$
\sigma_{i, j}\left(A^{\prime}\right)=\left\{\begin{array}{lr}
\sigma_{i, j}(A)+1 & \text { if } p \leq i<l \text { and } f \leq j<g \\
\sigma_{i, j}(A) & \text { otherwise } .
\end{array}\right.
$$

So, $A^{\prime} \prec_{B} A$.

Proposition 1 Let $A, C \in \mathcal{A}(R, S)$ such that $C$ covers $A$ in the Bruhat order. Let $p, f, g$ and $l$ be integers, with $1 \leq p<l \leq m$, and $1 \leq f<g \leq n$, such that

$$
A[\{p, l\} ;\{f, g\}]=I_{2},
$$

and, for any $(r, c) \in\{p, \ldots, l-1\} \times\{f, \ldots, g-1\}$,

$$
\sigma_{r c}(A)>\sigma_{r c}(C) \text {. }
$$

Then $A \preceq \widehat{B} C$.

Proof The $I_{2} \rightarrow L_{2}$ interchange that replaces $A[\{p, l\} ;\{f, g\}]=I_{2}$ with $L_{2}$ results in a matrix $D$ with $A \prec_{\widehat{B}} D$. By Lemma 3 , for any $(r, c) \in\{1, \ldots, m\} \times$ $\{1, \ldots, n\}$,

$$
\sigma_{r c}(D)=\left\{\begin{array}{ll}
\sigma_{r c}(A)-1 & \text { if }(r, c) \in\{p, \ldots, l-1\} \times\{f, \ldots, g-1\} \\
\sigma_{r c}(A) & \text { otherwise, }
\end{array} .\right.
$$

Since

$$
\sigma_{r c}(A)>\sigma_{r c}(C),
$$

for any $(r, c) \in\{p, \ldots, l-1\} \times\{f, \ldots, g-1\}$, and $A \prec_{B} C$, we conclude that $A \prec_{B} D \preceq_{B} C$. Using the fact that $C$ covers $A$ for the Bruhat order, we get $D=C$, and then $A \prec \widehat{B} C$.

Proposition 2 Let $A=\left[a_{i j}\right]$ and $C=\left[c_{i j}\right]$ be matrices in $\mathcal{A}(R, S)$ with $A \prec_{B} C$, and let $i$ and $j$ be integers with $i>1, j>1, a_{i j} \geq c_{i j}$,

$$
\sigma_{i j}(A)=\sigma_{i j}(C), \text { and } \sigma_{i-1, j}(A)>\sigma_{i-1, j}(C) .
$$

Then $\sigma_{i-1, j-1}(A)>\sigma_{i-1, j-1}(C)$. 
Proof Suppose that that $\sigma_{i-1, j-1}(A)=\sigma_{i-1, j-1}(C)$.

We have

$$
\sigma_{i, j}(A)=a_{i j}+\sigma_{i-1, j}(A)+\sigma_{i, j-1}(A)-\sigma_{i-1, j-1}(A),
$$

and similarly

$$
\sigma_{i, j}(C)=c_{i j}+\sigma_{i-1, j}(C)+\sigma_{i, j-1}(C)-\sigma_{i-1, j-1}(C) .
$$

Using hypothesis,

$$
\left(\sigma_{i-1, j}(C)-\sigma_{i-1, j}(A)\right)+\left(\sigma_{i, j-1}(C)-\sigma_{i, j-1}(A)\right) \geq 0 .
$$

As $\sigma_{i-1, j}(A)>\sigma_{i-1, j}(C)$ we conclude that $\sigma_{i, j-1}(C)>\sigma_{i, j-1}(A)$, a contradiction. Therefore, $\sigma_{i-1, j-1}(A)>\sigma_{i-1, j-1}(C)$.

With similar proof, we obtain the next result.

Proposition 3 Let $A=\left[a_{i j}\right]$ and $C=\left[c_{i j}\right]$ be matrices in $\mathcal{A}(R, S)$ with $A \prec_{B} C$, and let $i$ and $j$ be integers with $i>1, j>1, a_{i j} \geq c_{i j}$,

$$
\sigma_{i j}(A)=\sigma_{i j}(C) \text { and } \sigma_{i, j-1}(A)>\sigma_{i, j-1}(C) .
$$

Then $\sigma_{i-1, j-1}(A)>\sigma_{i-1, j-1}(C)$.

Proposition 4 Let $A=\left[a_{i j}\right]$ and $C=\left[c_{i j}\right]$ be matrices in $\mathcal{A}(R, S)$ with $A \prec{ }_{B} C$, and let $i$ and $j$ be integers with $i>1, a_{i j} \geq c_{i j}$,

$$
\sigma_{i j}(A)=\sigma_{i j}(C) \text {, and } \sigma_{i-1, j}(A)>\sigma_{i-1, j}(C) .
$$

Then $j>1$.

Proof Suppose that $j=1$. Since $\sigma_{i-1, j}(A)>\sigma_{i-1, j}(C)$ we have

$$
\sigma_{i j}(A)=a_{i j}+\sigma_{i-1, j}(A)>a_{i j}+\sigma_{i-1, j}(C) \geq c_{i j}+\sigma_{i-1, j}(C)=\sigma_{i, j}(C) .
$$

Consequently,

$$
\sigma_{i, j}(A)>\sigma_{i, j}(C)
$$

a contradiction. So, $j>1$.

With a similar proof, we obtain the following result.

Proposition 5 Let $A=\left[a_{i j}\right]$ and $C=\left[c_{i j}\right]$ be matrices in $\mathcal{A}(R, S)$ with $A \prec_{B} C$, and let $i$ and $j$ be integers with $j>1, a_{i j} \geq c_{i j}$,

$$
\sigma_{i j}(A)=\sigma_{i j}(C), \text { and } \sigma_{i, j-1}(A)>\sigma_{i, j-1}(C) .
$$

Then $i>1$. 


\section{The class $\mathcal{A}(n, 3)$}

In [4] an example evolving three matrices of $\mathcal{A}(6,3)$ shows the no coincidence of the Bruhat order and the Secondary Bruhat order in this class. The example is the following:

Example 1 Consider the three matrices

$$
A=\left[\begin{array}{llllll}
1 & 0 & 0 & 0 & 1 & 1 \\
1 & 0 & 1 & 1 & 0 & 0 \\
1 & 1 & 0 & 1 & 0 & 0 \\
0 & 0 & 0 & 1 & 1 & 1 \\
0 & 1 & 1 & 0 & 1 & 0 \\
0 & 1 & 1 & 0 & 0 & 1
\end{array}\right], \quad C=\left[\begin{array}{llllll}
0 & 0 & 0 & 1 & 1 & 1 \\
1 & 0 & 1 & 1 & 0 & 0 \\
1 & 1 & 0 & 1 & 0 & 0 \\
1 & 0 & 0 & 0 & 1 & 1 \\
0 & 1 & 1 & 0 & 1 & 0 \\
0 & 1 & 1 & 0 & 0 & 1
\end{array}\right]
$$

and

$$
D=\left[\begin{array}{llllll}
0 & 0 & 0 & 1 & 1 & 1 \\
1 & 1 & 0 & 1 & 0 & 0 \\
1 & 0 & 1 & 1 & 0 & 0 \\
1 & 0 & 0 & 0 & 1 & 1 \\
0 & 1 & 1 & 0 & 1 & 0 \\
0 & 1 & 1 & 0 & 0 & 1
\end{array}\right]
$$

A calculation shows that

$$
\Sigma_{A}>\Sigma_{D}>\Sigma_{C}
$$

Thus,

$$
A \prec_{B} D \prec_{B} C .
$$

Using the cover relation (see Theorem 3.1 in [4]) we know that $C$ covers $A$ and $D$ in the Secondary Bruhat order. This implies that $D$ and $A$ are incomparable in the Secondary Bruhat order.

In the next results we show that the two Bruhat orders coincide on $\mathcal{A}(n, 3)$, when $n \in\{3,4,5\}$.

Proposition 6 Let $R=\left(r_{1}, \ldots, r_{m}\right)$, and $S=\left(s_{1}, \ldots, s_{n}\right)$ be two nonincreasing positive integral vectors such that $\mathcal{A}(R, S) \neq \emptyset$. Let $U=\left(n-r_{m}, \ldots, n-r_{1}\right)$, and $Q=\left(m-s_{n}, \ldots, m-s_{1}\right)$. If the Bruhat order and the Secondary Bruhat order coincide in $\mathcal{A}(R, S)$, then these two orders coincide in $\mathcal{A}(U, Q)$.

Proof Let $A$ and $C$ be two matrices in $\mathcal{A}(U, Q)$. We know that if $A \prec \widehat{B} C$, then $A \prec_{B} C$. Suppose that $A \prec_{B} C$. Then for any $(r, c) \in\{1, \ldots, m\} \times$ $\{1, \ldots, n\}, \sigma_{r c}(A) \geq \sigma_{r c}(C)$ and there is $(i, j) \in\{1, \ldots, m\} \times\{1, \ldots, n\}$ such that $\sigma_{i j}(A)>\sigma_{i j}(C)$.

Let $D=\left[d_{i, j}\right]=L_{m}(J-A) L_{n}$ and $E=L_{m}(J-C) L_{n}$. If $(p, q) \in$ $\{1, \ldots, m\} \times\{1, \ldots, n\}$, then 


$$
\begin{aligned}
\sigma_{p q}(D) & =\sum_{g=1}^{p} \sum_{h=1}^{q} d_{g h} \\
& =p q-\sum_{g=m-p+1}^{m} \sum_{h=n-q+1}^{n} a_{g h} \\
& =p q-\left(r_{m-p+1}+\ldots+r_{m}\right)+s_{1}+\ldots+s_{n-q}-\sigma_{m-p, n-q}(A) \\
& \leq p q-\left(r_{m-p+1}+\ldots+r_{m}\right)+s_{1}+\ldots+s_{n-q}-\sigma_{m-p, n-q}(C) \\
& =\sigma_{p q}(E) .
\end{aligned}
$$

Since $\sigma_{i j}(A)>\sigma_{i j}(C)$ we get $\sigma_{m-i, n-j}(E)>\sigma_{m-i, n-j}(D)$. Therefore, $E \prec_{B} D$. Using the fact that $D, E \in \mathcal{A}(R, S)$, and the hypothesis, we have $E \prec \widehat{B} D$. Consequently, $D$ can be transformed into $E$ by a sequence of $L_{2} \rightarrow I_{2}$ interchanges.

If $P \in \mathcal{A}(U, Q)$ and $P[\{a, b\} ;\{c, d\}]=L_{2}$, then

$$
\left(L_{m} P L_{n}\right)[\{m-b+1, m-a+1\} ;\{n-d+1, n-c+1\}]=L_{2} .
$$

This implies that

$$
\left(J-L_{m} P L_{n}\right)[\{m-b+1, m-a+1\} ;\{n-d+1, n-c+1\}]=I_{2} .
$$

So, $C$ can be transformed into $A$ by a sequence of $L_{2} \rightarrow I_{2}$ interchanges. Thus, $A \prec \widehat{B} C$.

Using last result and the fact that the two Bruhat orders coincide on $\mathcal{A}(n, 0), \mathcal{A}(n, 1), \mathcal{A}(n, 2)$ we conclude the next result.

Corollary 1 The Bruhat order and the Secondary Bruhat order coincide on $\mathcal{A}(3,3), \mathcal{A}(4,3), \mathcal{A}(5,3)$.

Using Example 1 we will show that the two Bruhat orders do not coincide on $\mathcal{A}(n, 3)$, for $n \geq 7$.

Let $A, C$ and $D$ be the matrices described in Example 1.

Let $X_{A}=A[\{1,2,3,4\} ;\{1,2,3,4\}], X_{C}=C[\{1,2,3,4\} ;\{1,2,3,4\}]$ and $X_{D}=D[\{1,2,3,4\} ;\{1,2,3,4\}]$.

Case $\mathcal{A}(7,3)$. If

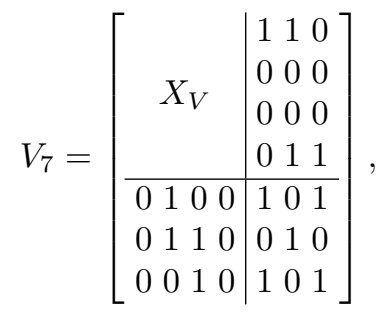

with $V \in\{A, C, D\}$ then, using similar arguments as in Example 1, we conclude that

$$
A_{7} \prec_{B} D_{7} \prec_{B} C_{7} .
$$

but $D_{7}$ and $A_{7}$ are incomparable in the Secondary Bruhat order. 
Case $\mathcal{A}(8,3)$. If

$$
V_{8}=\left[\begin{array}{lll|lllll}
X_{V} & & & & 0 & 0 & 0 & 0 \\
& & & 0 & 0 & 0 & 0 \\
& & 0 & 1 & 1 \\
\hline 0 & 1 & 0 & 0 & 1 & 1 & 0 & 0 \\
0 & 1 & 0 & 0 & 1 & 1 & 0 & 0 \\
0 & 0 & 1 & 0 & 0 & 0 & 1 & 1 \\
0 & 0 & 1 & 0 & 0 & 0 & 1 & 1
\end{array}\right],
$$

with $V \in\{A, C, D\}$ then, using similar arguments as in Example 1, we conclude that

$$
A_{8} \prec_{B} D_{8} \prec_{B} C_{8} .
$$

but $D_{8}$ and $A_{8}$ are incomparable in the Secondary Bruhat order.

Case $\mathcal{A}(\mathbf{n}, \mathbf{3})$, with $n \geq 9$. If

$$
V_{n}=\left[\begin{array}{l|l}
V & 0 \\
\hline 0 & G
\end{array}\right],
$$

with $V \in\{A, C, D\}$ and $G \in \mathcal{A}(n-6,3)$ then, using similar arguments as in Example 1, we conclude that

$$
A_{n} \prec_{B} D_{n} \prec_{B} C_{n} .
$$

but $D_{n}$ and $A_{n}$ are incomparable in the Secondary Bruhat order.

4 The class $\mathcal{A}(R, S)$, with $R=\left(2^{m}\right)$

In this section, we show that the Bruhat order and the Secondary Bruhat order coincide on $\mathcal{A}(R, S)$ with $R=\left(1^{m}\right)$ or $R=\left(2^{m}\right)$, as they do on $\mathcal{A}(n, 2)$.

Theorem 2 Let $R=\left(2^{m}\right)$, and $S=\left(s_{1}, \ldots, s_{n}\right)$ be two nonincreasing positive integral vectors such that $\mathcal{A}(R, S) \neq \emptyset$. Then the Bruhat order and the Secondary Bruhat order coincide on $\mathcal{A}(R, S)$.

Proof Let $A$ and $C$ be matrices in $\mathcal{A}(R, S)$. We know that $A \prec_{\widehat{B}} C$ implies that $A \prec_{B} C$. So we need to prove that if $A \prec_{B} C$, then $A \prec_{\widehat{B}} C$. It suffices to show this when $C$ covers $A$. So, from now on we assume that $C$ covers $A$ by the Bruhat order.

The strategy is to find integers $p, f, g$ and $l$, with $1 \leq p<l \leq m$, and $1 \leq f<g \leq n$, such that

$$
A[\{p, l\} ;\{f, g\}]=I_{2},
$$

and for any $(r, c) \in\{p, \ldots, l-1\} \times\{f, \ldots, g-1\}$,

$$
\sigma_{r c}(A)>\sigma_{r c}(C) \text {. }
$$


If this happens, then using Proposition 1 we get $A \prec_{\widehat{B}} C$.

Since $A \prec_{B} C$, there is a position $(i, j)$ such that $a_{i j}=1$ and $\sigma_{i j}(A)>$ $\sigma_{i j}(C)$ (the lexicographically first position where $\Sigma(A)$ and $\Sigma(C)$ differ, verifies). We choose such a position $(i, j)$ with $i+j$ as large as possible.

Applying Lemma 1 , we choose $\left(i_{0}, j_{0}\right) \in\{i+1, \ldots, m\} \times\{j+1, \ldots, n\}$ such that $a_{i_{0} j_{0}}=1$, and

for any $(r, c) \in\left\{i, \ldots, i_{0}-1\right\} \times\left\{j, \ldots, j_{0}-1\right\}, \quad \sigma_{r c}(A)>\sigma_{r c}(C)$.

We choose such a position $\left(i_{0}, j_{0}\right)$ with $i_{0}+j_{0}$ as small as possible. We consider three cases.

Case 1: $a_{i_{0} j}=a_{i j_{0}}=0$.

Then

$$
A\left[\left\{i, i_{0}\right\} ;\left\{j, j_{0}\right\}\right]=\left[\begin{array}{ll}
1 & 0 \\
0 & 1
\end{array}\right]=I_{2},
$$

and

$$
\text { for any }(r, c) \in\left\{i, \ldots, i_{0}-1\right\} \times\left\{j, \ldots, j_{0}-1\right\}, \quad \sigma_{r c}(A)>\sigma_{r c}(C) .
$$

Case 2: $a_{i_{0} j}=1$.

Because $i_{0}>i$ and $a_{i_{0}, j}=1$, by the maximality condition on $i+j$, we know that $\sigma_{i_{0}, j}(A)=\sigma_{i_{0}, j}(C)$. We also know that $\sigma_{i_{0}-1, j}(A)>\sigma_{i_{0}-1, j}(C)$. Moreover, we have $a_{i_{0}, j}=1 \geq c_{i_{0}, j}$ and $i_{0}>i \geq 1$. So, by Propositions 4 and 2 we have $j>1$, and $\sigma_{i_{0}-1, j-1}(A)>\sigma_{i_{0}-1, j-1}(C)$.

Applying Lemma 2, there is $\left(i_{1}, j_{1}\right) \in\left\{1, \ldots, i_{0}-1\right\} \times\{1, \ldots, j-1\}$ such that $a_{i_{1}, j_{1}}=1$ and

for any $(r, c) \in\left\{i_{1}, \ldots, i_{0}-1\right\} \times\left\{j_{1}, \ldots, j-1\right\}, \quad \sigma_{r, c}(A)>\sigma_{r, c}(C)$.

We choose such a position $\left(i_{1}, j_{1}\right)$ with $i_{1}+j_{1}$ as large as possible. We now consider three subcases.

Subcase 2.1: $i_{1}=i$.

Then

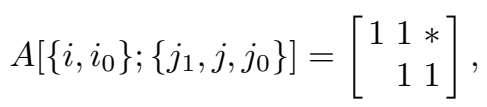

where $*$ denotes an unspecified entry.

Since any row of $A$ contains exactly two 1 's, we have $a_{i j_{0}}=0$ and $a_{i_{0} j_{1}}=0$. Thus, $A\left[\left\{i, i_{0}\right\} ;\left\{j_{1}, j_{0}\right\}\right]=I_{2}$ and

for any $(r, c) \in\left\{i, \ldots, i_{0}-1\right\} \times\left\{j_{1}, \ldots, j_{0}-1\right\}, \quad \sigma_{r, c}(A)>\sigma_{r, c}(C)$.

Subcase 2.2: $i_{1}>i$.

Then

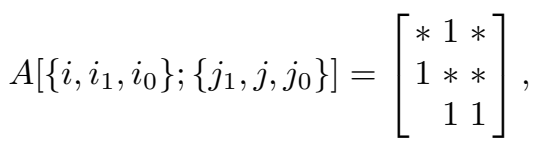


where $*$ denotes an unspecified entry.

Since any row of $A$ contains exactly two 1 's, we have $a_{i_{0} j_{1}}=0$.

If $a_{i_{1} j}=0$, then $A\left[\left\{i_{1}, i_{0}\right\} ;\left\{j_{1}, j\right\}\right]=I_{2}$ and

for any $(r, c) \in\left\{i_{1}, \ldots, i_{0}-1\right\} \times\left\{j_{1}, \ldots, j-1\right\}, \quad \sigma_{r, c}(A)>\sigma_{r, c}(C)$.

If $a_{i_{1} j}=1$, then

$$
A\left[\left\{i, i_{1}, i_{0}\right\} ;\left\{j_{1}, j, j_{0}\right\}\right]=\left[\begin{array}{ccc}
* & 1 & * \\
1 & 1 & * \\
0 & 1 & 1
\end{array}\right],
$$

where $*$ denotes an unspecified entry.

Consequently, $a_{i_{1} j_{0}}=0$ and $A\left[\left\{i_{1}, i_{0}\right\} ;\left\{j_{1}, j_{0}\right\}\right]=I_{2}$. Since

$$
\text { for any }(r, c) \in\left\{i, \ldots, i_{0}-1\right\} \times\left\{j, \ldots, j_{0}-1\right\}, \quad \sigma_{r c}(A)>\sigma_{r c}(C)
$$

and

$$
\text { for any }(r, c) \in\left\{i_{1}, \ldots, i_{0}-1\right\} \times\left\{j_{1}, \ldots, j-1\right\}, \quad \sigma_{r, c}(A)>\sigma_{r, c}(C),
$$

we obtain

for any $(r, c) \in\left\{i_{1}, \ldots, i_{0}-1\right\} \times\left\{j_{1}, \ldots, j_{0}-1\right\}, \quad \sigma_{r, c}(A)>\sigma_{r, c}(C)$.

Subcase 2.3: $i_{1}<i$.

Then

$$
A\left[\left\{i_{1}, i, i_{0}\right\} ;\left\{j_{1}, j, j_{0}\right\}\right]=\left[\begin{array}{r}
1 * * \\
1 * \\
1
\end{array}\right],
$$

where $*$ denotes an unspecified entry.

Since any row of $A$ contains exactly two 1 's, then $a_{i_{0} j_{1}}=0$. We consider three cases.

Subcase 2.3.1: $a_{i_{1} j}=0$.

Then $A\left[\left\{i_{1}, i_{0}\right\} ;\left\{j_{1}, j\right\}\right]=I_{2}$ and

$$
\text { for any }(r, c) \in\left\{i_{1}, \ldots, i_{0}-1\right\} \times\left\{j_{1}, \ldots, j-1\right\}, \quad \sigma_{r, c}(A)>\sigma_{r, c}(C) \text {. }
$$

Subcase 2.3.2: $a_{i_{1} j}=1$, and $\sigma_{i_{1} j}(A)>\sigma_{i_{1} j}(C)$.

Then

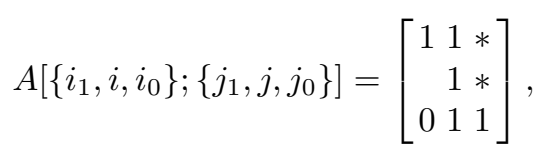

where $*$ denotes an unspecified entry.

Applying Lemma 1 , there is $\left(i_{2}, j_{2}\right) \in\left\{i_{1}+1, \ldots, m\right\} \times\{j+1, \ldots, n\}$ such that $a_{i_{2} j_{2}}=1$ and

$$
\text { for any }(r, c) \in\left\{i_{1}, \ldots, i_{2}-1\right\} \times\left\{j, \ldots, j_{2}-1\right\}, \quad \sigma_{r c}(A)>\sigma_{r c}(C) \text {. }
$$


Since $\sigma_{i_{0} j}(A)=\sigma_{i_{0} j}(C)$, we conclude that $i_{2} \leq i_{0}$. We have already identified two positions in row $i_{1}$ of $A$ that are occupied by 1 's, $\left(i_{1}, j_{1}\right)$ and $\left(i_{1}, j\right)$, then $a_{i_{1} j_{2}}=0$.

If $a_{i_{2} j}=0$, then $i_{2} \neq i_{0}$ and

$$
A\left[\left\{i_{1}, i_{2}, i_{0}\right\} ;\left\{j_{1}, j, j_{2}\right\}\right]=\left[\begin{array}{rrr}
1 & 1 & 0 \\
* & 1 \\
0 & 1 & *
\end{array}\right],
$$

where $*$ denotes an unspecified entry. Thus, $A\left[\left\{i_{1}, i_{2}\right\} ;\left\{j, j_{2}\right\}\right]=I_{2}$ and

$$
\text { for any }(r, c) \in\left\{i_{1}, \ldots, i_{2}-1\right\} \times\left\{j, \ldots, j_{2}-1\right\}, \quad \sigma_{r, c}(A)>\sigma_{r, c}(C) .
$$

If $a_{i_{2} j}=1$, then $a_{i_{2} j_{1}}=0$ and

$$
A\left[\left\{i_{1}, i_{2}\right\} ;\left\{j_{1}, j, j_{2}\right\}\right]=\left[\begin{array}{lll}
1 & 1 & 0 \\
0 & 1 & 1
\end{array}\right] .
$$

Thus, $A\left[\left\{i_{1}, i_{2}\right\} ;\left\{j_{1}, j_{2}\right\}\right]=I_{2}$.

Since $i_{2} \leq i_{0}$,

$$
\text { for any }(r, c) \in\left\{i_{1}, \ldots, i-1\right\} \times\left\{j_{1}, \ldots, j_{0}-1\right\}, \quad \sigma_{r, c}(A)>\sigma_{r, c}(C)
$$

and

for any $(r, c) \in\left\{i_{1}, \ldots, i_{2}-1\right\} \times\left\{j, \ldots, j_{2}-1\right\}, \quad \sigma_{r, c}(A)>\sigma_{r, c}(C)$,

we get

for any $(r, c) \in\left\{i_{1}, \ldots, i_{2}-1\right\} \times\left\{j_{1}, \ldots, j_{2}-1\right\}, \quad \sigma_{r, c}(A)>\sigma_{r, c}(C)$.

Subcase 2.3.3: $a_{i_{1} j}=1$ and $\sigma_{i_{1} j}(A)=\sigma_{i_{1} j}(C)$.

Then

$$
A\left[\left\{i_{1}, i, i_{0}\right\} ;\left\{j_{1}, j, j_{0}\right\}\right]=\left[\begin{array}{rrr}
1 & 1 & * \\
& 1 & * \\
0 & 1 & 1
\end{array}\right],
$$

where $*$ denotes an unspecified entry.

Since $j>1$ and $\sigma_{i_{1}, j-1}(A)>\sigma_{i_{1}, j-1}(C)$, by Proposition 5 , we get $i_{1}>1$. Using Proposition 3, we get $\sigma_{i_{1}-1, j-1}(A)>\sigma_{i_{1}-1, j-1}(C)$.

Applying Lemma 2, there is $\left(i_{3}, j_{3}\right) \in\left\{1, \ldots, i_{1}-1\right\} \times\{1, \ldots, j-1\}$ such that $a_{i_{3}, j_{3}}=1$ and

for any $(r, c) \in\left\{i_{3}, \ldots, i_{1}-1\right\} \times\left\{j_{3}, \ldots, j-1\right\}, \quad \sigma_{r, c}(A)>\sigma_{r, c}(C)$.

Then

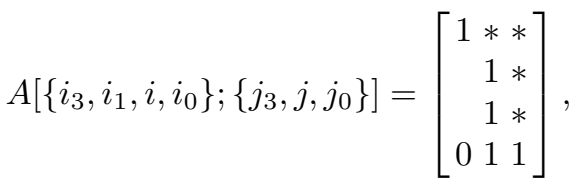


where $*$ denotes an unspecified entry.

If $a_{i_{3} j}=0$ and $j_{3} \geq j_{1}$, then

$$
A\left[\left\{i_{3}, i_{1}, i, i_{0}\right\} ;\left\{j_{1}, j_{3}, j, j_{0}\right\}\right]=\left[\begin{array}{rrrr}
* & 1 & 0 & * \\
1 & * & 1 & * \\
* & 1 & * \\
0 & 1 & 1
\end{array}\right]
$$

where $*$ denotes an unspecified entry. Thus, $A\left[\left\{i_{3}, i_{0}\right\} ;\left\{j_{3}, j\right\}\right]=I_{2}$.

Since

$$
\text { for any }(r, c) \in\left\{i_{1}, \ldots, i_{0}-1\right\} \times\left\{j_{1}, \ldots, j-1\right\}, \quad \sigma_{r, c}(A)>\sigma_{r, c}(C)
$$

and

for any $(r, c) \in\left\{i_{3}, \ldots, i_{1}-1\right\} \times\left\{j_{3}, \ldots, j-1\right\}, \quad \sigma_{r, c}(A)>\sigma_{r, c}(C)$,

we have

$$
\text { for any }(r, c) \in\left\{i_{3}, \ldots, i_{0}-1\right\} \times\left\{j_{3}, \ldots, j-1\right\}, \quad \sigma_{r, c}(A)>\sigma_{r, c}(C) \text {. }
$$

If $a_{i_{3} j}=0$, and $j_{3}<j_{1}$, then

$$
A\left[\left\{i_{3}, i_{1}, i, i_{0}\right\} ;\left\{j_{3}, j_{1}, j, j_{0}\right\}\right]=\left[\begin{array}{rrrr}
1 & * & 0 & * \\
0 & 1 & 1 & * \\
* & 1 & * \\
0 & * & 1 & 1
\end{array}\right]
$$

where $*$ denotes an unspecified entry. Consequently, $A\left[\left\{i_{3}, i_{1}\right\} ;\left\{j_{3}, j\right\}\right]=I_{2}$ and

for any $(r, c) \in\left\{i_{3}, \ldots, i_{1}-1\right\} \times\left\{j_{3}, \ldots, j-1\right\}, \quad \sigma_{r, c}(A)>\sigma_{r, c}(C)$.

If $a_{i_{3} j}=1$, and $\sigma_{i_{3} j}(A)>\sigma_{i_{3} j}(C)$, then

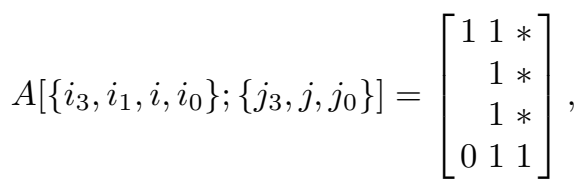

where $*$ denotes an unspecified entry. So, we argue as in Subcase 2.3.2, with rows $i_{3}, i, i_{0}$ and columns $j_{3}, j, j_{0}$.

If $a_{i_{3} j}=1$ and $\sigma_{i_{3} j}(A)=\sigma_{i_{3} j}(C)$, then we repeat this Subcase 2.3.3, with rows $i_{3}, i, i_{0}$ and columns $j_{3}, j, j_{0}$. This process ends because $A$ has a finite number of rows.

Case 3: $a_{i j_{0}}=1$ and $a_{i_{0} j}=0$.

Because $j_{0}>j$ and $a_{i, j_{0}}=1$, by the maximality condition on $i+j$, we know that $\sigma_{i, j_{0}}(A)=\sigma_{i, j_{0}}(C)$. We also know that $\sigma_{i, j_{0}-1}(A)>\sigma_{i, j_{0}-1}(C)$. 
Moreover, we have $a_{i, j_{0}}=1 \geq c_{i, j_{0}}$ and $j_{0}>j \geq 1$. So, by Propositions 5 and 3 we have $i>1$ and $\sigma_{i-1, j_{0}-1}(A)>\sigma_{i-1, j_{0}-1}(C)$.

Applying Lemma 2 , there is $\left(i_{4}, j_{4}\right) \in\{1, \ldots, i-1\} \times\left\{1, \ldots, j_{0}-1\right\}$ such that $a_{i_{4}, j_{4}}=1$ and

for any $(r, c) \in\left\{i_{4}, \ldots, i-1\right\} \times\left\{j_{4}, \ldots, j_{0}-1\right\}, \quad \sigma_{r, c}(A)>\sigma_{r, c}(C)$.

We now consider three subcases.

Subcase 3.1: $j_{4}=j$.

Then

$$
A\left[\left\{i_{4}, i, i_{0}\right\} ;\left\{j, j_{0}\right\}\right]=\left[\begin{array}{ll}
1 & * \\
1 & 1 \\
0 & 1
\end{array}\right],
$$

where $*$ denotes an unspecified entry. We consider three cases.

Subcase 3.1.1: $a_{i_{4} j_{0}}=0$.

Then $A\left[\left\{i_{4}, i_{0}\right\} ;\left\{j, j_{0}\right\}\right]=I_{2}$. Using the fact that

$$
\text { for any }(r, c) \in\left\{i, \ldots, i_{0}-1\right\} \times\left\{j, \ldots, j_{0}-1\right\}, \quad \sigma_{r, c}(A)>\sigma_{r, c}(C),
$$

and

for any $(r, c) \in\left\{i_{4}, \ldots, i-1\right\} \times\left\{j_{4}, \ldots, j_{0}-1\right\}, \quad \sigma_{r, c}(A)>\sigma_{r, c}(C)$

we obtain

for any $(r, c) \in\left\{i_{4}, \ldots, i_{0}-1\right\} \times\left\{j_{4}, \ldots, j_{0}-1\right\}, \quad \sigma_{r, c}(A)>\sigma_{r, c}(C)$.

Subcase 3.1.2: $a_{i_{4} j_{0}}=1$ and $\sigma_{i_{4} j_{0}}(A)>\sigma_{i_{4} j_{0}}(C)$.

Then

$$
A\left[\left\{i_{4}, i, i_{0}\right\} ;\left\{j, j_{0}\right\}\right]=\left[\begin{array}{ll}
1 & 1 \\
1 & 1 \\
0 & 1
\end{array}\right] .
$$

Applying Lemma 1 , there is $\left(i_{5}, j_{5}\right) \in\left\{i_{4}+1, \ldots, m\right\} \times\left\{j_{0}+1, \ldots, n\right\}$ such that $a_{i_{5} j_{5}}=1$ and

for any $(r, c) \in\left\{i_{4}, \ldots, i_{5}-1\right\} \times\left\{j_{0}, \ldots, j_{5}-1\right\}, \quad \sigma_{r c}(A)>\sigma_{r c}(C)$.

We claim that $i_{5} \leq i$. Suppose that $i_{5}>i$. Then $\sigma_{i, j_{0}}(A)>\sigma_{i, j_{0}}(C)$. Using the maximality condition on $i+j$ we get a contradiction. So, $i_{5} \leq i$.

Since any row of $A$ contains exactly two 1 's, we have $a_{i_{4} j_{5}}=0$ and $i_{5} \neq i$. Moreover, $a_{i_{5} j_{4}}=0$ or $a_{i_{5} j}=0$.

If $a_{i_{5} j_{4}}=0$, then

$$
A\left[\left\{i_{4}, i_{5}\right\} ;\left\{j_{4}, j_{0}, j_{5}\right\}\right]=\left[\begin{array}{ccc}
1 & 1 & 0 \\
0 & * & 1
\end{array}\right],
$$

where $*$ denotes an unspecified entry. Consequently, $A\left[\left\{i_{4}, i_{5}\right\} ;\left\{j_{4}, j_{5}\right\}\right]=I_{2}$. 
Since

for any $(r, c) \in\left\{i_{4}, \ldots, i-1\right\} \times\left\{j_{4}, \ldots, j_{0}-1\right\}, \quad \sigma_{r, c}(A)>\sigma_{r, c}(C)$

and

for any $(r, c) \in\left\{i_{4}, \ldots, i_{5}-1\right\} \times\left\{j_{0}, \ldots, j_{5}-1\right\}, \quad \sigma_{r c}(A)>\sigma_{r c}(C)$,

we obtain

for any $(r, c) \in\left\{i_{4}, \ldots, i_{5}-1\right\} \times\left\{j_{4}, \ldots, j_{5}-1\right\}, \quad \sigma_{r c}(A)>\sigma_{r c}(C)$.

As

If $a_{i_{5} j_{4}}=1$, then $a_{i_{5} j_{0}}=0$

$$
A\left[\left\{i_{4}, i_{5}\right\} ;\left\{j_{4}, j_{0}, j_{5}\right\}\right]=\left[\begin{array}{ccc}
1 & 1 & 0 \\
1 & 0 & 1
\end{array}\right] .
$$

Consequently, $A\left[\left\{i_{4}, i_{5}\right\} ;\left\{j_{0}, j_{5}\right\}\right]=I_{2}$ and

for any $(r, c) \in\left\{i_{4}, \ldots, i_{5}-1\right\} \times\left\{j_{0}, \ldots, j_{5}-1\right\}, \quad \sigma_{r c}(A)>\sigma_{r c}(C)$.

Subcase 3.1.3: $a_{i_{4} j_{0}}=1$ and $\sigma_{i_{4} j_{0}}(A)=\sigma_{i_{4} j_{0}}(C)$.

Then

$$
A\left[\left\{i_{4}, i, i_{0}\right\} ;\left\{j, j_{0}\right\}\right]=\left[\begin{array}{ll}
1 & 1 \\
1 & 1 \\
0 & 1
\end{array}\right] .
$$

Since $j_{0}>1$ and $\sigma_{i_{4}, j_{0}-1}(A)>\sigma_{i_{4}, j_{0}-1}(C)$, by Proposition 5 , we get $i_{4}>1$. Using Proposition 3, we get $\sigma_{i_{4}-1, j_{0}-1}(A)>\sigma_{i_{4}-1, j_{4}-1}(C)$.

Applying Lemma 2 , there is $\left(i_{6}, j_{6}\right) \in\left\{1, \ldots, i_{4}-1\right\} \times\left\{1, \ldots, j_{0}-1\right\}$ such that $a_{i_{6}, j_{6}}=1$ and

for any $(r, c) \in\left\{i_{6}, \ldots, i_{4}-1\right\} \times\left\{j_{6}, \ldots, j_{0}-1\right\}, \quad \sigma_{r, c}(A)>\sigma_{r, c}(C)$.

We consider three cases.

Subcase 3.1.3.1: $j_{6}=j$. We argue as Case 3.1.

Subcase 3.1.3.2: $j_{6}>j$. We argue as Case 3.2.

Subcase 3.1.3.3: $j_{6}<j$. We argue as Case 3.3.

This process ends because $A$ has a finite number of rows.

Subcase 3.2: $j_{4}>j$.

Then

$$
A\left[\left\{i_{4}, i, i_{0}\right\} ;\left\{j, j_{4}, j_{0}\right\}\right]=\left[\begin{array}{ccc}
* & 1 & * \\
1 & * & 1 \\
0 & * & 1
\end{array}\right],
$$

where $*$ denotes an unspecified entry.

Since any row of $A$ contains exactly two 1 's, we have $a_{i j_{4}}=0$. We consider three cases. 
Subcase 3.2.1: $a_{i_{4} j_{0}}=0$.

Then

$$
A\left[\left\{i_{4}, i, i_{0}\right\} ;\left\{j, j_{4}, j_{0}\right\}\right]=\left[\begin{array}{ccc}
* & 1 & 0 \\
1 & 0 & 1 \\
0 & * & 1
\end{array}\right],
$$

where $*$ denotes an unspecified entry. Consequently, $A\left[\left\{i_{4}, i\right\} ;\left\{j_{4}, j_{0}\right\}\right]=I_{2}$ and

for any $(r, c) \in\left\{i_{4}, \ldots, i-1\right\} \times\left\{j_{4}, \ldots, j_{0}-1\right\}, \quad \sigma_{r, c}(A)>\sigma_{r, c}(C)$.

Subcase 3.2.2: $a_{i_{4} j_{0}}=1$ and $\sigma_{i_{4} j_{0}}(A)>\sigma_{i_{4} j_{0}}(C)$.

Then

$$
A\left[\left\{i_{4}, i, i_{0}\right\} ;\left\{j, j_{4}, j_{0}\right\}\right]=\left[\begin{array}{ccc}
* & 1 & 1 \\
1 & 0 & 1 \\
0 & * & 1
\end{array}\right],
$$

where $*$ denotes an unspecified entry.

Applying Lemma 1 , there is $\left(i_{7}, j_{7}\right) \in\left\{i_{4}+1, \ldots, m\right\} \times\left\{j_{0}+1, \ldots, n\right\}$ such that $a_{i_{7} j_{7}}=1$ and

for any $(r, c) \in\left\{i_{4}, \ldots, i_{7}-1\right\} \times\left\{j_{0}, \ldots, j_{7}-1\right\}, \quad \sigma_{r c}(A)>\sigma_{r c}(C)$.

We claim that $i_{7} \leq i$. Suppose that $i_{7}>i$. Then $\sigma_{i, j_{0}}(A)>\sigma_{i, j_{0}}(C)$. Using the maximality condition on $i+j$ we get a contradiction. So, $i_{7} \leq i$.

Since any row of $A$ contains exactly two 1 's, we have $a_{i_{4} j_{7}}=0$ and $i_{7} \neq i$. Moreover, $a_{i_{7} j_{4}}=0$ or $a_{i_{7} j}=0$.

If $a_{i_{7} j_{4}}=0$, then

$$
A\left[\left\{i_{4}, i_{7}\right\} ;\left\{j_{4}, j_{0}, j_{7}\right\}\right]=\left[\begin{array}{ccc}
1 & 1 & 0 \\
0 & * & 1
\end{array}\right],
$$

where $*$ denotes an unspecified entry. Consequently, $A\left[\left\{i_{4}, i_{7}\right\} ;\left\{j_{4}, j_{7}\right\}\right]=I_{2}$.

Since

for any $(r, c) \in\left\{i_{4}, \ldots, i-1\right\} \times\left\{j_{4}, \ldots, j_{0}-1\right\}, \quad \sigma_{r, c}(A)>\sigma_{r, c}(C)$

and

for any $(r, c) \in\left\{i_{4}, \ldots, i_{7}-1\right\} \times\left\{j_{0}, \ldots, j_{7}-1\right\}, \quad \sigma_{r c}(A)>\sigma_{r c}(C)$,

we obtain

for any $(r, c) \in\left\{i_{4}, \ldots, i_{7}-1\right\} \times\left\{j_{4}, \ldots, j_{7}-1\right\}, \quad \sigma_{r c}(A)>\sigma_{r c}(C)$.

If $a_{i_{7} j_{4}}=1$, then $a_{i_{7} j_{0}}=0$ and

$$
A\left[\left\{i_{4}, i_{7}\right\} ;\left\{j_{4}, j_{0}, j_{7}\right\}\right]=\left[\begin{array}{ccc}
1 & 1 & 0 \\
1 & 0 & 1
\end{array}\right]
$$


where $*$ denotes an unspecified entry. Consequently, $A\left[\left\{i_{4}, i_{7}\right\} ;\left\{j_{0}, j_{7}\right\}\right]=I_{2}$ and

for any $(r, c) \in\left\{i_{4}, \ldots, i_{7}-1\right\} \times\left\{j_{0}, \ldots, j_{7}-1\right\}, \quad \sigma_{r c}(A)>\sigma_{r c}(C)$.

Subcase 3.2.3: $a_{i_{4} j_{0}}=1$ and $\sigma_{i_{4} j_{0}}(A)=\sigma_{i_{4} j_{0}}(C)$.

Then

$$
A\left[\left\{i_{4}, i, i_{0}\right\} ;\left\{j, j_{4}, j_{0}\right\}\right]=\left[\begin{array}{lll}
* & 1 & 1 \\
1 & 0 & 1 \\
0 & * & 1
\end{array}\right],
$$

where $*$ denotes an unspecified entry.

Since $j_{0}>1$ and $\sigma_{i_{4}, j_{0}-1}(A)>\sigma_{i_{4}, j_{0}-1}(C)$, by Proposition 5 , we get $i_{4}>1$. Using Proposition 3, we get $\sigma_{i_{4}-1, j_{0}-1}(A)>\sigma_{i_{4}-1, j_{4}-1}(C)$.

Applying Lemma 2 , there is $\left(i_{8}, j_{8}\right) \in\left\{1, \ldots, i_{4}-1\right\} \times\left\{1, \ldots, j_{0}-1\right\}$ such that $a_{i_{8}, j_{8}}=1$ and

for any $(r, c) \in\left\{i_{8}, \ldots, i_{4}-1\right\} \times\left\{j_{8}, \ldots, j_{0}-1\right\}, \quad \sigma_{r, c}(A)>\sigma_{r, c}(C)$.

We consider three cases

Subcase 3.2.3.1: $j_{8}=j$. We argue as Case 3.1.

Subcase 3.2.3.2: $j_{8}>j$. We argue as Case 3.2.

Subcase 3.2.3.3: $j_{8}<j$. We argue as Case 3.3.

This process ends because $A$ has a finite number of rows.

Subcase 3.3: $j_{4}<j$.

Then

$$
A\left[\left\{i_{4}, i, i_{0}\right\} ;\left\{j_{4}, j, j_{0}\right\}\right]=\left[\begin{array}{rrr}
1 & * & * \\
1 & 1 \\
0 & 1
\end{array}\right],
$$

where $*$ denotes an unspecified entry.

Since any row of $A$ contains exactly two 1 's, we have $a_{i j_{4}}=0$. Moreover, $a_{i_{4} j}=0$ or $a_{i_{4} j_{0}}=0$.

If $a_{i_{4} j}=0$, then $A\left[\left\{i_{4}, i\right\} ;\left\{j_{4}, j\right\}\right]=I_{2}$ and

for any $(r, c) \in\left\{i_{4}, \ldots, i-1\right\} \times\left\{j_{4}, \ldots, j-1\right\}, \quad \sigma_{r, c}(A)>\sigma_{r, c}(C)$.

If $a_{i_{4} j_{0}}=0$, then $A\left[\left\{i_{4}, i\right\} ;\left\{j_{4}, j_{0}\right\}\right]=I_{2}$ and

for any $(r, c) \in\left\{i_{4}, \ldots, i-1\right\} \times\left\{j_{4}, \ldots, j_{0}-1\right\}, \quad \sigma_{r, c}(A)>\sigma_{r, c}(C)$.

Corollary 2 Let $R=\left(1^{m}\right)$ and $S=\left(s_{1}, \ldots, s_{n}\right)$ be two nonincreasing positive integral vectors such that $\mathcal{A}(R, S) \neq \emptyset$. Then the Bruhat order and the Secondary Bruhat order coincide on $\mathcal{A}(R, S)$. 
Proof Let $A, C \in \mathcal{A}(R, S)$ such that $A \preceq{ }_{B} C$. Let $D$ be the $m$-by- $n+1$ matrix such that the first column has all entries equal to one and removing the first column we have the matrix $A$. Similarly, let $E$ be the $m$-by- $n+1$ matrix such that the first column has all entries equal to one and removing the first column we have the matrix $C$. Then $D$ and $E$ are matrices in $\mathcal{A}(U, V)$ with $U=\left(2^{m}\right)$ and $V=\left(m, s_{1}, \ldots, s_{n}\right)$. Since $A \preceq_{B} C$ we get $D \preceq_{B} E$. By last theorem, $D \preceq_{\widehat{B}} E$. Thus, as the first columns of $D$ and $E$ have all entries equal to one, $A \preceq \widehat{B} C$.

\section{Conclusions}

We studied the coincidence of the two extensions of the classical Bruhat order on $S_{n}$, stated by Bruadi and Hwang in [3]. We proved the coincidence of these two orders in $\mathcal{A}(R, S)$ occurs when $R=\left(2^{m}\right)$ or $R=\left(1^{m}\right)$.

Using the transpose of matrices we obtain the two next results.

Theorem 3 Let $S=\left(2^{n}\right)$, and $R=\left(r_{1}, \ldots, r_{m}\right)$ be two nonincreasing positive integral vectors such that $\mathcal{A}(R, S) \neq \emptyset$. Then the Bruhat order and the Secondary Bruhat order coincide on $\mathcal{A}(R, S)$.

Corollary 3 Let $S=\left(1^{m}\right)$ and $R=\left(r_{1}, \ldots, r_{m}\right)$ be two nonincreasing positive integral vectors such that $\mathcal{A}(R, S) \neq \emptyset$. Then the Bruhat order and the Secondary Bruhat order coincide on $\mathcal{A}(R, S)$.

By Proposition 6 the reader know that there are many classes of matrices where the two Bruhat orders coincide, for instance $\mathcal{A}(12,11)$.

We observed that if $R=\left(n^{p}, 2^{m-p}\right)$, with $0 \leq p \leq m$ and $S=\left(s_{1}, \ldots, s_{n}\right)$ are two nonincreasing positive integral vectors such that $\mathcal{A}(R, S) \neq \emptyset$ then the two Bruhat orders coincide on $\mathcal{A}(R, S)$. An interesting problem is the characterization of other classes of matrices where the two Bruhat orders coincide. Other important problem is to find properties of these two partial orders. It is well known that the classical Bruhat order in $S_{n}$ is Eulerian and lexicographically shellable (see [19] and [12]). It is interesting to know if, at least in these classes, the Bruhat order retains these properties.

\section{References}

1. R.A. Brualdi, Matrices of zeros and ones with fixed row and column-sum vectors, Linear Algebra Appl., 33 (1980) 159-231.

2. R.A. Brualdi, Combinatorial Matrix Classes, Encyclopedia of Mathematics and its Applications, vol. 108: Cambridge University Press, Cambridge (2006).

3. R.A. Brualdi and S.-G. Hwang, A Bruhat order for the class of $(0,1)$-matrices with row sum vector $R$ and column sum vector $S$, Electronic Journal of Linear Algebra, 12 (2004) $6-16$.

4. R.A. Brualdi and L. Deaett, More on the Bruhat order for (0,1)-matrices, Linear algebra and its Applications, 421 (2007) 219-232. 
5. R.A. Brualdi and E. Fritscher, Bruhat order of tournaments, Linear Algebra and its Applications, 458 (2014) 261-279.

6. R.A. Brualdi and G. Dahl, Doubly stochastic matrices and the Bruhat order, Czechoslovak Mathematical Journal, 66 (141) (2016) 681-700.

7. R. A. Brualdi and M. W.Schroeder, Alternating sign matrices and their Bruhat order, Discrete Mathematics, 340, Issue 8 (2017) 1996-2019.

8. R.A. Brualdi, R. Fernandes and S. Furtado, On the Bruhat Order of Labeled Graphs, Discrete Applied Mathematics, 258 (2019), 49-64.

9. A. Conflitti, C.M. Fonseca and R. Mamede, The maximal lenght of a chain in the Bruhat order for a class of binary matrices,Linear algebra and its Applications, 436 (2012), 753757

10. A. Conflitti, C.M. da Fonseca, R. Mamede, On the largest size of an antichain in the Bruhat order for A(2k,k), Order, 30 (2013), 255-260

11. H.F. Cruz, R. Fernandes and S. Furtado, Minimal matrices in the Bruhat order for symmetric (0,1)-matrices, Linear Algebra and its Applications, 530 (2017) 160-184.

12. P. H. Edelman, The Bruhat Order of the Symmetric Group is Lexicographically Shellable, Proceedings of the American Mathematical Society, 82, (1981) 355-358

13. R. Fernandes, H.F. Cruz and D. Salomão, Latin Squares and their Bruhat Order, submitted.

14. D. Gale, A theorem on flows in networks, Pacific Journal of Mathematics, 7 (1957) 1073-1082.

15. M. Ghebleh, On maximum chains in the Bruhat order of $\mathcal{A}(n, 2)$,Linear algebra and its Applications, 446 (2014), 377-387.

16. M. Ghebleh, Antichains on $(0,1)$-matrices through inversions, Linear algebra and its Applications, 458 (2014), 503-511

17. H.J. Ryser, Combinatorial properties of matrices of zeros and ones. Canadian Journal of Mathematics, 9 (1957), 371-377.

18. R.P. Stanley, Enumerative Combinatorics, vol. 2, Cambridge Studies in Advanced Mathematics, vol. 62, Cambdrige University Press, Cambridge (1999).

19. D. N. Verma, Möbius inversion for the Bruhat ordering on a Weyl group, Annales scientiques de l.N.S., tome 4, no 3 (1971), 393-398 